\title{
A strengths-based case management service for people with serious mental illness in Israel: A randomized controlled trial
}

Marc Gelkopf ${ }^{\mathrm{a}, \mathrm{b}}$, Liron Lapid ${ }^{\mathrm{a}, \mathrm{b}^{*}}$, Nomi Werbeloff ${ }^{\mathrm{c}}$, Stephen Levine, ${ }^{\mathrm{a}}$, Yaara ZismanIlani $^{\mathrm{a}, \mathrm{b}}$, David Roe ${ }^{\mathrm{a}, \mathrm{b}}$

${ }^{a}$ Department of Community Mental Health, Faculty of Social Welfare and Health Sciences, University of Haifa, Israel.

${ }^{\mathrm{b}}$ Center for Community Mental Health, Research, Practice and Policy, Faculty of Social Welfare and Health Sciences, University of Haifa, Israel.

${ }^{\mathrm{c}}$ Division of Psychiatry, University College London, England.

*Corresponding Author: Liron Lapid, Department of Community Mental Health, Faculty of Social Welfare and Health Sciences, University of Haifa, Mount Carmel, Haifa, 3190501, Israel. Telephone: +972-3-6429018, email: lapid.liron@gmail.com. 


\section{A strengths-based case management service for people with serious mental illness in Israel: A randomized controlled trial}

\section{Highlights}

- There is paucity of controlled and randomized studies assessing case management services for people with serious mental illness (SMI).

- A strengths-based case management (SBCM) service is assessed in 1276 individuals with SMI, using a randomized controlled approach.

- SBCM participants improved in self-efficacy, unmet needs, and general quality of life, and set more goals than the control group.

- SBCM services are effective in helping individuals with SMI set personal goals and use psychiatric rehabilitation services in a better and more focused manner. 


\title{
A strengths-based case management service for people with serious mental illness in Israel: A randomized controlled trial
}

\begin{abstract}
Case management services for people with serious mental illness are generally found to be effective, but controlled and randomized studies assessing such services are scarce. The aim of the present study was to assess the effectiveness of a new strengths-based case management (SBCM) service in Israel, using a randomized controlled approach. The sample consisted of 1276 individuals with serious mental illness, who consume psychiatric rehabilitation services (PRS) in the community, and were randomly assigned to receive or not to receive the SBCM service in addition to treatment-as-usual PRS. Quality of life, goal setting and attainment, unmet needs, self-efficacy, interpersonal relationships, symptom severity, and service utilization were assessed by clients at onset and after 20 months. Results show that SBCM participants improved in self-efficacy, unmet needs, and general quality of life, and set more goals than the control group. SBCM participants also consumed fewer services at follow-up. Results suggest that SBCM services are effective in helping individuals with serious mental illness set personal goals and use PRS in a better and more focused manner.
\end{abstract}

Keywords: strengths-based model; psychiatric rehabilitation services; outcome assessment 


\section{A strengths-based case management service for people with serious mental illness in Israel: A randomized controlled trial}

\section{Introduction}

Case management for people with serious mental illness can be described as a "process or method for insuring that the consumer is provided needed services in a coordinated, effective and efficient manner" (Pratt et al., 2007). The need for case management services originally arose out of the process of deinstitutionalization, through which the locus of treatment for people with serious mental illness moved from the hospital to the community (Mueser et al., 1998; Pratt et al., 2007). This required them to take a more active role in the selection and coordination of mental health services, a role with which they often need assistance (Mueser et al., 1998; Pratt et al., 2007). Case management services are designed to assess needs, create and carry out a service plan to help meet those needs, and monitor progress, in order to improve community participation and avoid relapse and re-hospitalization (Backus et al., 2008; Pratt et al., 2007).

The use of case management services has been steadily associated with a host of positive outcomes. These include reduced relapse rates, reduced hospital time, increased use of community services, reduced symptomatology, improved functioning, and improved quality of life (Bedell et al., 2000; Burns et al., 2007; Chamberlain and Rapp, 1991; Dietrich et al., 2011; Mueser et al., 1998; O’Brien et al., 2012; Ziguras and Stuart, 2000). A number of case management models currently exist, including broker case management (Intagliata, 1982), the clinical model (Kanter, 2010), the rehabilitation model (Anthony et al., 1993), assertive community treatment, sometimes referred to as intensive case management (Dietrich et al., 2011), and the strengths model for case management (Rapp and Goscha, 2006, 2008). 
The latter model, unlike the common deficit-oriented, illness-focused approach, takes a goal-oriented approach that focuses on the clients' strengths and emphasizes their capacity for growth and recovery (Fukui et al., 2012; Rapp and Goscha, 2006, 2008). The strengths model emphasizes the importance of using and acquiring naturally existing resources in the community, and perceives the client as the one to lead his/her rehabilitation process (Rapp and Goscha, 2006, 2008). This reflects the growing emphasis in mental health on recovery from serious mental illness (Anthony, 1993; Deegan, 1988), which, in the rehabilitation process, translates into focusing on personal goals and the assessment of functioning and skills training (Anthony et al., 1988; Mueser et al., 1998).

Recent evidence suggests that people who receive strengths-based case management (SBCM) are hospitalized less frequently, are more independent in daily life, achieve more goals, function better in the competitive employment and educational domains, report greater social support, and have overall better physical and mental health (Barry et al., 2003; Fukui et al., 2012; Rapp and Goscha, 2006). A recent meta-analysis reviewing five experimental and quasi-experimental studies concluded that SBCM might not be better than other models of service delivery in improving quality of life or functioning (Ibrahim et al., 2014), but since none of the studies reviewed have monitored fidelity, those results are not unequivocally comparable to previous evidence (e.g., Fukui et al., 2012). Nonetheless, more sound additional evidence is required regarding the effects of SBCM.

In Israel, a milestone in the development of psychiatric rehabilitation services was the approval of the Rehabilitation of the Mentally Disabled in the Community Law (RMD) in the year 2000. The legislation specified a set of psychiatric rehabilitation services to be provided to people with serious mental illness. Those 
services aim to address key needs, and include case management services to facilitate optimal use of the services to meet those needs. While services in areas such as employment, education, or housing were rapidly developed and extensively consumed (Aviram et al., 2012; Roe et al., 2010a), the development and implementation of a systematic case management service lagged behind, even though the need for such a service was previously recommended (Aviram, 2010; Aviram et al., 2012; Roe et al., 2010a).

In response, a recent effort has been directed to develop and implement a strengths-based case management (SBCM) service, inspired by the strengths model (Rapp and Goscha, 2006, 2008) and the values of recovery (Anthony, 1993), and adapted to the administrative and cultural background of mental health services in Israel. The aim of this service is to promote active engagement of clients both in defining and attaining goals, mainly through the selection and support of recoveryrelevant community resources (see Box 1 for a full description).

Studies assessing the effectiveness of case management services, and particularly strengths-based case management, typically use non-experimental or quasi-experimental methods (Fukui et al., 2012; Ibrahim et al., 2014), and there is a need for more methodologically sound studies. The current study uses a randomized and controlled design to assess the impact of the SBCM service on client outcomes, including goal setting and attainment, quality of life, interpersonal relationships, unmet needs, self-efficacy, symptom severity, and service utilization.

\section{Methods}

\subsection{Setting and design}

The current study was conducted in the central and northern regions of Israel between January 2008 and March 2012. The study was performed by comparing 
individuals receiving the newly established strengths-based case management service in addition to regular psychiatric rehabilitation services (PRS) (SBCM-PRS - the experimental group) and individuals receiving only treatment-as-usual PRS (TAUPRS - the control group). Clients' self-assessments were collected at two time-points, 20 months apart. In addition, demographic (including diagnosis) and service utilization data were obtained from the Rehabilitation Mental Health Registry (RMHR), a national registry which includes data from all individuals who have received mental health rehabilitation services from the Israeli Ministry of Health.

\subsection{Procedure and randomization}

Participants were randomly sampled from the overall pool of all individuals either receiving or about to receive PRS. Eligibility for PRS, being a public system of services, is having being diagnosed with a mental illness, which has caused at least a $40 \%$ psychiatric disability as determined by a medical committee, composed of a psychiatrist and recognized by the National Insurance regulations. Previous research has estimated that the majority $(86 \%)$ of those who met these criteria had a diagnosis of a psychotic-related disorder (Struch et al., 2011). Additional inclusion criteria were fluency in Hebrew and sufficient competence to provide informed consent. Exclusion criteria were current hospitalization or currently being acutely psychotic or violent.

We continually received, from the Ministry of Health, a complete and updated (anonymous) list of individuals, who were current or future clients of PRS in the northern and central regions of Israel $(n=\sim 10,000)$. When informed of new openings in the SBCM service, we regularly drew potential participants from this list in a randomized way, i.e., recruitment was ongoing. To obtain a first randomization procedure, we applied a simple computer-based randomization algorithm, which produced a list of potential participants, and ensured representative sampling by using 
a criteria matrix of age and service dependence (i.e., the level of care the person requires). Potential participants were then approached by Ministry of Health workers, and if they agreed to participate in the study and met the inclusion criteria, were approached by the research team interviewers (within about a week), who administered the questionnaires, and recorded information for follow-up purposes. Participants provided informed consent to participate in the study and to authorize the use of data from the RMHR. The interviews were conducted in person, took $20-40$ minutes each, and the participants were paid NIS 35 (\$10) for their time. After filling in the baseline questionnaire, individuals were randomly assigned (by coin-tossing) to the SBCM-PRS experimental group or the PRS only treatment-as-usual (TAU-PRS) control group. A follow-up assessment was performed approximately 20 months after baseline. Design and procedure for the current study were approved by the ethics committee of the University of Haifa.

\subsection{Participants}

Figure 1 describes the flow of the 1545 clients participating in the various stages of the study, of whom $59.8 \%(n=919)$ were male. The mean age was 39.2 ( $\mathrm{SD}=12.6)$. Of all participants, $917(64.5 \%)$ were single, $260(18.3 \%)$ were divorced or separated, $221(15.5 \%)$ were married. 838 participants (66. \%) were born in Israel. Most participants $(n=878,65.7 \%)$ had 12 years or less of education. Most $(n=774$, $81 \%$ ) were diagnosed with schizophrenia, or schizoaffective or other psychotic disorders. Univariate analysis, comparing participants who completed the follow-up assessment with those who dropped out across main demographic and outcome measures at baseline, showed no difference between the groups. (Analysis is available from the corresponding author.) Additionally, a chi-square analysis comparing the number of dropouts across groups showed that control group participants dropped out 
of the study significantly more than experimental group participants, namely $58.4 \%$ of those who dropped out were in the control group $\left(\chi_{(1)}^{2}=14.84 ; p<.001\right)$.

-Insert Figure 1 about here-

\subsection{Description of the strengths-based case management (SBCM) intervention and} treatment as usual (TAU)

The objectives, core values, and guidelines of the strengths-based case management service (SBCM) assessed in the current study, as well as details regarding the role of the case manager, training and supervision, and adaptations to the cultural and organizational context in Israel, are described in Box 1. The psychiatric rehabilitation services (PRS), i.e., the treatment-as-usual intervention, are described in Box 2, including objectives, guiding principles, and rehabilitation domains.

-Insert Boxes 1 and 2 about here-

\subsection{Fidelity assessment of the SBCM intervention}

A fidelity assessment scale was created to assess adherence of the SBCM service to key components of the present model. The scale includes five domains: (1) the rehabilitation alliance, (2) advocating, mediating, and collaborating with various elements in the client's life, (3) use of rehabilitation tools, (4) the rehabilitation plan, and (5) the case manager's work environment. Each domain includes 4-6 items rated on a 5-point Likert scale (1-5). Ratings are performed by clients, case managers, the service's director, and professional consultants. For the purpose of this study, ratings were calculated by compounding and averaging a sample of 50 clients, 27 case managers, the service's director and local professional consultants, as well as a sample of 30 case files. Results showed the SBCM service to have satisfactory fidelity $(\mathrm{M}=3.69, \mathrm{SD}=0.5)$. Two domains, the rehabilitation alliance, and the CM's work 
environment rated 4.4 and 4.3, respectively. No domain rated less than 3.05 (Roe et al., 2013).

\subsection{Measures}

The study tools were constructed after a comprehensive review of existing instruments assessing outcomes in mental health services. Different items were extracted from a variety of tools to provide a multidimensional outcome assessment of the current service, and were then adapted to the local cultural and organizational context. Both extraction of items and adaptation were performed by a committee of different stakeholders, including clients. A pilot study was initiated to assess face validity and reliability of the tools within the Israeli context (Roe et al., 2010b). A two-week test-retest on a sample of 30 clients of PRS services was found to have a Pearson correlation of $\mathrm{r}=.45(\mathrm{p}<.001)$.

\subsubsection{Clients' self-assessment}

Self-assessment included the following measures.

Quality of life was assessed using eight items in six domains, originally based on the Manchester Short Assessment of Quality of Life scale (MANSA, Priebe et al., 1999). The quality of life domains were as follows: (1) employment (one item), (2) income (one item), (3) housing (two items), (4) leisure (one item), (5) physical activity (two items), and (6) studies (one item). Each domain evaluated clients' subjective quality of life on a 4-point Likert scale (1="very unsatisfied" to 4="very satisfied"), e.g., "How satisfied are you with your work?" "How satisfied are you with your housing conditions?" The full scale showed modest internal consistency (Cronbach's alpha $=0.68)$.

Interpersonal relationships and the social domain: Participants assessed the subjective quality of various types of relationships in their lives (e.g., close family, 
friends, spouse, children) and the social aspect of their lives in general, e.g., "How satisfied are you with the way you get along with people?" "How satisfied are you with your relationship with your immediate family?" Seven items on a 4-point Likert scale (1="very unsatisfied" to 4="very satisfied") were included in this measure, which was also based on the Manchester Short Assessment of Quality of Life scale (MANSA, Priebe et al., 1999). The full scale showed acceptable internal consistency $($ Cronbach's alpha $=0.75)$.

Unmet needs: Participants assessed the degree to which their choices and desires, i.e., their current needs in several domains, were being met, using a tool constructed by the research team based on recent literature (e.g., Wiersma et al., 2009). This tool included seven items in six domains: employment (two items, for those who are/are not employed), income, social life, leisure; study, and housing (one item each). For each domain, participants were asked to rate the degree to which the current situation matched their wishes and choices, on a 4-point Likert scale $(1=$ "does not match at all" to 4="matches very much"), e.g., "To what degree do you feel your housing situation matches your wishes and choices?" The scale showed reasonable internal consistency (Cronbach's alpha $=0.64)$.

Self-efficacy was assessed using a 12-item scale designed by the research team, based on recent literature (e.g., Chen et al., 2001; Mancini, 2007; McCann et al., 2008). Participants were asked to assess, on a 5-point Likert scale, to what extent they believed in their ability to achieve progress in various domains, such as employment, relationships, social life, or community belongingness (e.g., "I'm optimistic and believe in my ability to make progress in the employment domain"). Internal consistency for this scale was high (Cronbach's alpha $=0.90)$. 
Goal setting and attainment: A tool containing three items assessing goal setting and goal attainment was created especially for this study, based on principles from previous work (Kiresuk and Sherman, 1968; Kiresuk et al. 1994). Participants reported the number of goals set during the year, their contents in brief, and assessed the attainment of each goal (not attained/partially attained/fully attained). The scale showed modest internal consistency (Cronbach's alpha for goals attained was 0.61). Two research assistants, independently and followed by a triangulation procedure, categorized responses to the goal contents item into one of the following domains: employment or study, housing, mental health, family and relationships, leisure, physical health, financial, and social life.

Psychiatric symptoms were assessed using the Colorado Symptom Index (CSI; Shern et al., 1994). The CSI is a brief 14-item self-report measure of psychiatric symptoms, in which respondents report the frequency of various symptoms they have experienced during the past month (e.g., "How often during the past month have you felt depressed?"). Each item is scored on a 5-point Likert scale (1=“not at all” to 5=“every day"). The full scale showed very good internal consistency (Cronbach's alpha $=0.92)$.

\subsubsection{Measures obtained from the Rehabilitation Mental Health Registry}

Data obtained from the RMHR included the following variables: psychiatric diagnosis, demographic information, i.e., gender, age, marital status, country of birth, and education, and utilization of psychiatric rehabilitation services (history and current use). Since the registry of services used typically takes a few months to stabilize after entering the system (e.g., individuals may be assigned to services but not use them, or start to use them a few months after assignment, or change them early on if deemed irrelevant), only data regarding "stably registered" participants, 
who had been assigned to services at least six months before the onset of the study, were included in the analysis.

\subsection{Data analysis}

We checked for differences in dependent variables across the experimental and control groups at baseline, using $t$-tests and Wilcoxon tests. For the main analysis, we used a multivariate analysis of covariance (MANCOVA) to compare the dependent variables across the experimental and control groups at follow-up, inserting quality of life at baseline, age, and gender as covariates. We then performed separate analyses of covariance (ANCOVA) for quality of life, unmet needs, symptom severity and selfefficacy with the same covariates as in the above mentioned MANCOVA. We used a Wilcoxon test to compare delta scores in non-parametric dependent variables, namely the two goal variables, interpersonal relationships, quality of life domains, unmet needs domains, and service utilization. We performed maximum likelihood missing value imputations except in quality of life domains, unmet needs domains, and goal attainment, when a response reflected the noted irrelevance of the item to the participant (e.g., does not study, did not set goals, etc.)

\section{Results}

\subsection{Comparison of the experimental and control groups before receiving the SBCM service}

Findings of univariate analysis comparing the experimental (SBCM-PRS) and control treatment-as-usual (TAU-PRS) group at baseline are presented in Table 1. Differences in demographic variables were found in gender and age, with more male and younger participants in the SBCM-PRS group. Several differences in main outcome variables at study onset were found, with the SBCM-PRS participants having 
a lower quality of life and subjectively worse interpersonal relationships, and attaining more goals.

-Insert Table 1 about here-

\subsection{Assessment of the intervention: MANCOVA, ANCOVAs, and non-parametric analyses comparing the SBCM-PRS and TAU-PRS groups}

To control for the difference found in gender, age, and quality of life between the experimental and control groups at the beginning of the study, a MANCOVA analysis was performed, with gender, age and baseline quality of life as covariates, and with the parametric self-report outcome measures, namely quality of life, unmet needs, symptom severity, and self-efficacy, as dependent variables. Results show an overall impact of the SBCM service on the self-report parametric outcome measures (Hotelling's Trace=.01; $\left.F_{(4,1242)}=3.90 ; p<.01\right)$. Significant effects were also found for gender (Hotelling's Trace $\left.=.009 ; F_{(4,1242)}=2.94 ; \quad p<.05\right)$, age (Hotelling's Trace=.09; $F_{(4,1242)}=26.83 ; p<.001$ ), and quality of life at baseline (Hotelling's Trace=.34; $\left.F_{(4,1242)}=104.68 ; p<.001\right)$.

As shown in Table 2, separate ANCOVAs revealed a significant improvement in quality of life, unmet needs and self-efficacy in SBCM-PRS participants.

-Insert Table 2 about here-

Results of the a-parametric analysis for the remainder of the self-report outcome measures, namely interpersonal relationships, goals set, goals attained, service utilization, separate quality of life domains, and separate unmet needs domains, are presented in Table 3. SBCM-PRS participants' satisfaction with their interpersonal relationships had decreased significantly less, and they set significantly more goals after receiving the service, compared to the TAU-PRS control group. Their quality of life had improved to a greater extent (or declined less) than participants in the control 
group in the physical health, leisure, housing and employment domains. TAU-PRS participants had attained significantly more goals. Additionally, the groups differed significantly in service utilization patterns, in that experimental group participants consumed fewer psychiatric rehabilitation services at follow-up compared to baseline, whereas control group participants' utilization of services remained the same. No differences were found in change scores between the experimental and control group in any of the unmet needs domains.

-Insert Table 3 about here-

To determine a difference in goal contents between the experimental and control groups at follow-up, a chi-square analysis was conducted for participants who set goals, for each of the eight goal domains. Results indicated a significant difference between the groups in the employment or study domain, in favor of the SBCM group. Of SBCM participants who set goals, $72.8 \%$ had an employment or study goal, compared to $63.3 \%$ in the control group $\left(\chi_{(1)}^{2}=9.99 ; p<.01\right)$. No differences were found between groups when comparing the remaining domains.

\section{Discussion}

The current study suggests that the SBCM service can be set up and implemented within an Israeli administrative and cultural setting, as was evident from the fidelity assessments. Furthermore, and most importantly, results show an overall positive impact of the SBCM service on participants' self-report outcomes in several domains. SBCM-PRS participants showed significant, albeit modest, increases in selfefficacy unmet needs, and overall quality of life, as well as in the quality of life domains of employment, leisure, housing, and physical health. They had also set significantly more goals. In addition, although a decline in participants' satisfaction of their interpersonal relationships was observed in both groups, the SBCM-PRS group 
showed significantly less decline. Conversely, it was found that the TAU-PRS control group attained significantly more goals than the SBCM-PRS group. Finally, although service utilization patterns remained the same in the TAU-PRS group, SBCM-PRS participants consumed fewer services at the end of the study. No other significant outcomes were found (i.e., no difference in unmet needs domains, symptom severity, and two quality of life domains).

Results regarding the current SBCM service correspond to the service's designated purpose of assisting people with serious mental illness living in the community to make progress toward their personal recovery, and demonstrate that the service is indeed beneficial for its clients. As a whole, results are in line with previous findings on case management interventions, which include improved quality of life, unmet needs and self-efficacy (Mueser et al., 1998; O'Brien et al., 2012; Ziguras and Stuart, 2000). Given that one of the goals of the SBCM service is to assist in selecting and utilizing rehabilitation services, the improved quality of life found in this study may be attributed to more appropriate choice and better use of such services. However, we do not have any specific data on the subject, and further research is needed to address this issue. Increased self-efficacy might reflect a heightened sense of control, hope, and belief in one's abilities, which are consistent with the principles of the strengths model, from which the current CM model is derived. We have also found that individuals that undergo SBCM have less unmet needs, which basically means better correspondence between wishes and choices and the specific situation the client is in, in relation to a specific life domain. These results lend credence to the importance of the basic premises of the model, namely the promotion through active engagement of clients in the process of both defining as well as bringing into play goal-directed behavior. 
The finding that SBCM-PRS participants set but did not attain more goals than TAU-PRS participants should be considered in light of current literature on setting and attaining goals within the recovery process. People with serious mental illness, who experienced many failures in their recovery process, often adopt an unmotivated "giving up" attitude as a defense mechanism against further disappointment (Deegan, 1996). For them, the very act of setting a personally meaningful goal, which is a special focus of the SBCM service, can be seen as a major achievement, because it often means that they are ready to risk a failure or delay in its attainment (Corrigan, 2011; Slade, 2009; Weingarten, 2005). Thus, failure to attain a goal does not necessarily mean failure in the recovery process. On the contrary, the readiness to take risks can be seen as a positive turning point in the process (Corrigan, 2011; Slade, 2009; Weingarten, 2005).

An additional explanation for this lower goal attainment rate might lie in the finding that SBCM-PRS participants set more goals related to employment or study. Such goals are typically difficult to attain as they depend not only on preliminary skills training but also on external factors (e.g., job availability), which cannot always be provided by the services themselves. Thus, the fact that SBCM-PRS participants have set more employment or study goals might have contributed to a lower goal attainment rate. Furthermore, as goal setting and attainment are central to the SBCM service, this might indicate a tendency for a more thorough and long-term approach toward goals, and thus a longer time-frame for goal attainment, as well as the possibility that for some clients, too many goals were set too soon.

The finding that SBCM-PRS participants consumed fewer services at follow-up and the TAU-PRS participants' service utilization remained the same is inconsistent with previous findings (Chamberlain and Rapp, 1991; Ziguras and Stuart, 2000). It is 
possible that the SBCM service aim of improving the use of rehabilitation services might initially mean becoming more selective, focused, and goal-oriented when choosing services, rather than simply using all available services.

The lack of significant results in the area of symptom severity might mirror the fact that the overall aims of PRS do not expressly include symptom reduction. They include illness management processes that might lead to better coping with symptoms (as witnessed by improvement on quality of life factors) but not actually to reducing them.

Results of the current study support the benefit of providing a case management service within a mental health system, and particularly as part of the ongoing psychiatric rehabilitation "basket of services" in Israel, which has already shown implementation success (Aviram et al., 2012; Roe et al., 2010a). Offering this service as part of the "basket of services" might become the policy change leading the system to its next level of development, in that it can contribute to improving the link between the individual's goals/needs and the variety of PRS. Thus, services would be viewed not in and of themselves, but as a means to assist people in their individual processes (Drake et al., 2011).

While considering these results, several limitations should be noted. First, it is difficult to generalize from the current setting to other service settings outside Israel, which might be organized differently and target different domains or populations. Second, some of the measures might lack good reliability because they were set up especially for this study. Third, since control group participants had higher quality of life at study onset, the results regarding the significant effect of the intervention on this domain might have been influenced by regression to the mean. Finally, it is 
noteworthy that many of the changes were modest, and hence, there is a need to assess the SBCM service over longer periods of time.

Future studies should continue to apply a randomized and controlled approach to assess the efficacy and efficiency of strengths-based case management. Furthermore, the generalizability of SBCM to other types of psychiatric rehabilitation settings and in other cultures should also be assessed. Another recommendation for future studies is to attempt to address the question of how treatment attrition is affected by SBCM. Finally, the specific impact of SBCM on the goal setting process should also be addressed in future studies.

In conclusion, in spite of its limitations, the current study, which is probably the largest existing controlled study on case management, offers additional evidence of the potential of this service to assist individuals during their recovery process, and specifically in using the psychiatric rehabilitation system in a way that fits their

personal goals. This service might be an important addendum to regular rehabilitation services, helping the individual choose and succeed in optimally exploiting those services. More specifically, adding the service to the current standard system in Israel might have benefits for clients and the system as a whole.

\section{Acknowledgement}

The establishment of the SBCM service and the current study were made possible by the Laszlo N. Tauber Family Foundation, through the Moshe Hess Association, and the support of the Rehabilitation department at the State of Israel Ministry of Health. The authors declare no conflict of interest in relation to the subject of this study. 


\section{References}

Anthony, W.A., Cohen, M., Farkas, M., Cohen, B.F., 1988. Clinical care update: The chronically mentally ill: Case management - more than a response to a dysfunctional system. Community Ment. Hlt. J. 24 (3), 219-228.

Anthony, W., Forbess, M., Cohen, R. 1993. Rehabilitation-oriented case management, in: Harris, M., Bergman, C. (Eds.), Case management for mental ill patients. Chord, Harwood Academic Publisher, pp. 99-118.

Anthony, W.A., 1993. Recovery from mental illness: The guiding vision of the mental health service system in the 1990s. Psychosoc. Rehabil. J. 16 (4), 11-23.

Aviram, U., 2010. Rehabilitation of the mentally disabled in the community law: Intermediate assessment and preparation for the future. Medicine: Psychiat. 14, 14-23. (in Hebrew).

Aviram, U., Ginath, Y., Roe, D., 2012. Israel's rehabilitation in the community of persons with mental disabilities law: Challenges and opportunities. Psychiatr. Serv. 63 (2), 110-112.

Backus, L., Weinkove, J., Lucas, M., Jespersen, S., 2008. Outcome of planned discharge from case management: A 3 year retrospective study. Australas.Psychiatry 16 (1), 33-38.

Barry, K.L., Zeber, J.E., Blow, F.C., Valenstein, M., 2003. Effects of the strengths model versus assertive community treatment model on participant outcomes and utilization: Two-year follow-up. Psychiatr. Rehabil. J. 26 (3), 268-277.

Bedell, J.R., Cohen, N.L., Sullivan, A., 2000. Case management: The current best practices and the next generation of innovation. Community Ment. Hlt. J.36 (2), 179-194.

Burns, T., Catty, J., Dash, M., Roberts, C., Lockwood, A., Marshall, M., 2007. Use of intensive case management to reduce time in hospital in people with severe mental illness: Systematic review and meta-regression. BMJ 335 (7615), 336-340.

Chamberlain, R., Rapp, C.A., 1991. A decade of case management: A methodological review of outcome research. Community Ment. Hlt. J. 27 (3), 171-188.

Chen, G., Gully, S., Eden, D., 2001. Validation of a new general self-efficacy scale. Organ. Res. Methods 4 (1), 62-83.

Corrigan, P.W., 2011. The dignity to fail. Psychiatr. Serv. 62 (3), 241.

Deegan, P.E., 1988. Recovery: The lived experience of rehabilitation. Psychosoc. Rehabil. J. 11 (4), 11-19.

Deegan, P.E., 1996. Recovery as a journey of the heart. Psychiatr. Rehabil. J. 19 (3), 91-97.

Dietrich, M., Irving, C.B., Park, B., Marshall, M., 2011. Intensive case management for people with severe mental illness. Cochrane Database of Systematic Reviews 10, Art. No.: CD007906. 
Drake, R.E., Hogan, M.F., Slade, M., Thornicroft, G., 2011. Editorial: Commentary on Israel's psychiatric rehabilitation law. Isr. J. Psychiatry Relat. Sci. 48 (4), 227-229.

Farkas, M., Soydan. A.S., Gagne, C., 2000. Introduction to Rehabilitation Readiness. Boston University, Center for Psychiatric Rehabilitation, Boston.

Fukui, S., Goscha, R., Rapp, C.A., Mabry, A., Liddy, P., Marty, D., 2012. Strengths model case management fidelity scores and client outcomes. Psychiatr. Serv. 63 (7), 708-710.

Ibrahim, N., Michail, M., Callaghan, P., 2014. The strengths based approach as a service delivery model for severe mental illness: A meta-analysis of clinical trials. BMC Psychiatry 14 (1), 243.

Intagliata, J., 1982. Improving the quality of community care for the chronically mentally disabled: The role of case management. Schizophr. Bull. 8 (4), 655674.

Kanter, J., 2010. Clinical case management, in: Brandell, J.R., (Ed.), Theory and Practice of Clinical Social Work ( $2^{\text {nd }}$ ed.). Sage Publications, Thousand Oaks CA, pp. 561-586.

Kiresuk, T.J., Sherman, R.E., 1968. Goal attainment scaling: A general method for evaluating comprehensive community mental health programs. Community Ment. Hlt. J. 4 (6), 443-453.

Kiresuk, T.J, Smith, A., Cardillo, J.E., 1994. Goal Attainment Scaling: Applications, Theory, and Measurement. Psychology Press, New York.

Mancini, M.A., 2007. The role of self-efficacy in recovery from serious psychiatric disabilities. Qual. Soc. Work 6 (1), 49-74.

McCann, T.V., Clark, E., Lu, S., 2008. The self-efficacy model of medication adherence in chronic mental illness. J. Clin. Nurs. 17 (11c), 329-340.

Mueser, K.T., Bond, G.R., Drake, R.E., Resnick, S.G., 1998. Models of community care for severe mental illness: A review of research on case management. Schizophr. Bull., 24 (1), 37-74.

Mueser, K.T., Noordsy, D.L., Drake, R.E., Fox, L., 2003. Motivational interviewing, in: Mueser, K.T., Noorsdy, D. L., Drake, R.E., Fox, L. (Eds.), Integrated Treatment for Dual Disorders: A Guide to Effective Practice. Guilford Press, New York, pp. 108-120.

O’Brien, S., McFarland, J., Kealy, B., Pullela, A., Saunders, J., Cullen, W., Meagher, D., 2012. A randomized-controlled trial of intensive case management emphasizing the recovery model among patients with severe and enduring mental illness. Ir. J. Med. Sci. 181 (3), 301-308.

Pratt, C.W., Gill, K.J., Barrett, N.M., Roberts, M.M., 2007. Assertive community treatment and case management, in: Pratt, C.W., Gill, K.J., Barrett, N.M., Roberts, M.M. (Eds.), Psychiatric Rehabilitation, second ed. Elsevier Academic Press, Burlington, MA, pp. 191-218. 
Priebe, S., Huxley, P., Knight, S., Evans, S., 1999. Application and results of the Manchester short assessment of quality of life (MANSA). Int. J. Soc. Psychiatry 45 (1), 7-12.

Rapp, C.A., Goscha, R.J., 2006. The purpose, principles and research results of the strengths model, in: Rapp, C.A., Goscha, R. (Eds.), The Strengths Model: Case Management with People with Psychiatric Disabilities, second ed. Oxford University Press, Oxford. pp. 54-73.

Rapp, C.A., Goscha, R.J., 2008. Strengths-based case management, in: Mueser, K.T., Jeste, D.V. (Eds.), Clinical Handbook of Schizophrenia. Guilford Press, New York, NY, pp. 319-328.

Rehabilitation of the Mentally Disabled in the Community Law, 2000. Jerusalem. Ministry of Justice, 2000. Available at: http://www.moital.gov.il/NR/exeres/E935CE19-2094-41E1-B26ED3AD28CA8DBB.htm

Roe, D., Gelkopf, M., Levine, S., Weinstein, Y., Telem, A., Haimovich, S., ZismanIlani, Y., 2013. Personal Psychiatric Rehabilitation Plans Coordinators: Presentation of Study Results. Report submitted to the Ministry of Health. [In Hebrew].

Roe, D., Lachman, M., Mueser, K.T., 2010. Editorial: A decade of the Israeli psychiatric rehabilitation law. Isr. J. Psychiatry Relat. Sci. 47 (3), 164-165.

Roe, D., Werbeloff, N., Gelkopf, M., 2010. Do persons with severe mental illness who consume the psychiatric rehabilitation basket of services in Israel have better outcomes than those who do not? Isr. J. Psychiatry Relat. Sci. 47(3), 166170.

Shern, D.L., Wilson, N.Z., Coen, A.S., Patrick, D.C., Foster, M., Bartsch, D.A., Demmler, J., 1994. Client outcomes II: Longitudinal client data from the Colorado treatment outcome study. Milbank Q. 72 (1), 123-148.

Slade, M., 2009. The contribution of risk-taking to recovery, in: Slade, M. (Eds.), Personal Recovery and Mental Illness: A Guide to Mental Health Professionals. Cambridge University Press, New York. pp. 176-181.

State of Israel Ministry of Health., 2015. Rehabilitation within the Community. Retrieved from: http://www.health.gov.il/English/Topics/Mental_Health/rehabilitation/Pages/def ault.aspx

Struch, N., Shereshevsky, Y., Naon, D., Fischman, N., 2011. The Contribution of Community Rehabilitation to Reducing Psychiatric Hospitalization in the Mental Health System. Myers-JDC-Brookdale Institute, Jerusalem, Israel. [in Hebrew].

University of Kansas, School of Social Welfare, Office of Mental Health Research and Training., n.d. Strengths-Based Case Management Fidelity Scale. Retrieved from: http://mentalhealth.socwel.ku.edu/fidelity-resources-0

Weingarten, R., 2005. Calculated risk-taking and other recovery processes for my psychiatric disability. Psychiatr. Rehabil. J. 29 (1), 77-80.

Wiersma, D., van den Brink, R., Wolters, K., McCabe, R., Bullenkamp, J., Hansson, L., Lauber, C., Martinez-Leal, R., Rossler, W., Salize, H., Bjorkman, T., Torres- 
Gonzales, F., Wright, D.J., Priebe, S., 2009. Individual unmet needs for care: Are they sensitive as outcome criterion for the effectiveness of mental health services interventions? Soc. Psychiatry Psychiatr. Epidemiol. 44 (4), 317-324.

Ziguras, S.J., Stuart, G.W., 2000. A meta-analysis of the effectiveness of mental health case management over 20 years. Psychiatr. Serv. 51 (11), 1410-1421.

- See Boxes 1 and 2 in the following page - 


\section{Box 1: The strengths-based case management (SBCM) intervention for individuals with severe mental illness in Israel}

\section{Objectives:}

1. Promote active engagement of clients in the process of defining and attaining personally meaningful goals.

2. Provide assistance in selecting and utilizing services and natural community resources which are most likely to help clients make progress toward their recovery goals.

\section{Core values/ beliefs:}

- Any person with severe mental illness can make progress toward recovery and achieve community integration.

- Clients can and should take an active role in their rehabilitation process, and set their own individually-defined goals.

\section{Guidelines for practice:}

1. Coordination between the client, the services he/she uses, and other relevant elements, which is essential for the effectiveness of the process.

2. Clients' personal goals are at the basis of the rehabilitation plan, which is jointly created by the client and the case manager.

3. SBCM are a means to promote clients' recovery process and the attainment of their goals.

4. Rehabilitation plans should be plain, clear, and measurable within a specific timeframe.

5. Progress depends on the support of all elements in the clients' environment, particularly the clients' family.

\section{Role of the case manager:}

1. Mediate between the client, the psychiatric rehabilitation services (PRS), and the Ministry of Health regional bureau (the principal provider and regulator).

2. Assist with adjustment to new PRS services and with changing services when necessary.

3. Promote the use and active participation of clients in PRS and SBCM services (i.e., outreach work).

4. Promote active engagement of clients in setting personal goals and creating a rehabilitation plan. 
Box 1 (cont'd.)

Training and supervision:

- Case managers are trained, experienced mental health professionals, who have undergone a 60-hour SBCM training program.

- Weekly individual and group supervision by trained professionals. Adaptations of the strengths model to the local and organizational context:

- Case manager responsibilities include an administrative function, e.g., placement of client into PRS, due to the service being a part of the national PRS system.

- Coordinative work with specific PRS providers such as integrated supported employment services.

- Supervision and group supervision include the incorporation of tools and skills from other models, such as rehabilitation readiness (Farkas et al., 2000) and motivational interviewing (Mueser et al., 2003), in addition to the strengths model tools and skills.

- Higher caseload ratio of 32:1 at the most. 
Box 2: Treatment-as-usual psychiatric rehabilitation services in Israel (TAU-PRS)

Description based on Ministry of Health website (Ministry of Health, 2015)

Objectives: Enable the rehabilitation and community integration of people with psychiatric disabilities, and improve their quality of life.

\section{Guiding principles:}

- Any person in any condition is capable of learning, development, and change.

- Rehabilitation services endorse a person-centered approach, and a belief in the individual's strengths and abilities.

- Rehabilitation services are based on a respectful, sharing, and optimistic approach, which sees the individual as a whole within the society and as having a potential worthy of realization.

- A tailor-made rehabilitation process according to individual needs, values, and ambitions.

- The rehabilitation work is based on developing the client's abilities at a suitable pace and level of difficulty, thus accumulating positive experiences in different life domains. Abilities are developed by means of direct and indirect guidance (e.g., modeling) and by exposing the client to various opportunities.

- Inclusion of family members and other close persons is helpful to the rehabilitation process.

- Hope is central to recovery-based rehabilitation.

\section{Rehabilitation domains (the "basket of services") and their objectives:}

1. Housing: improve competencies for independent living in the community and provide housing facilities where necessary.

2. Employment: improve competencies and help the client obtain employment according to the client's abilities and wishes.

3. Education: support clients in fulfilling their academic abilities and complete their education according to their abilities and wishes.

4. Leisure and social life: assist clients in acquiring social skills, facilitating a sense of belonging and reducing loneliness, making connections and achieving community integration.

5. Case management (coordination of treatment).

6. Family support: support and consult clients' families and reduce practical and emotional burden.

7. Dental care: provide financial support.

Service providers: PRS are provided by trained mental health professionals

*Although this domain is listed as a rehabilitation service, in practice, the only such service currently available is the strengths-based case management described in the current study. 
Table 1: Demographics and outcome measures at onset for the experimental and control group

\begin{tabular}{ccc}
$\begin{array}{c}\text { SBCM-PRS } \\
(\mathbf{N}=\mathbf{8 0 8})\end{array}$ & $\begin{array}{c}\text { TAU-PRS } \\
(\mathbf{N}=\mathbf{7 3 7})\end{array}$ & Univariate statistics \\
\cline { 1 - 2 } $\mathrm{M}(\mathrm{SD}) / \mathrm{N}(\%)$ & $\mathrm{M}(\mathrm{SD}) / \mathrm{N}(\%)$ &
\end{tabular}

\section{Demographics}

Gender

Male

Female

Age

Marital status

Single

Married/lives with a partner

Separated/divorced

Widowed

Country of birth

Israel

Other

Education

12 years or less

High school diploma

Professional training/academic

Yeshiva/other

Diagnosis

Schizophrenia, schizoaffective and

psychotic disorders

Mood disorder (MDD/Bipolar)

Anxiety disorder

Personality disorder

Other

\section{Service user variables}

Quality of life (1-4)

Unmet needs (1-4)

Symptom severity (1-5)

Self-efficacy (1-5)

Interpersonal relationships (1-4)

Goals set (0-3)

$507(62.83 \%)$

$\chi^{2}=6.34 ; \mathrm{p}<.05$

$300(37.17 \%)$

$412(56.52 \%)$

37 (Mdn)

$317(43.48 \%)$

$\begin{array}{ll}37 \text { (Mdn) } & 39 \text { (Mdn) } \\ 521(65.53 \%) & 396(63.26 \%) \\ 119(14.97 \%) & 102(16.29 \%) \\ 146(18.36 \%) & 114(18.21 \%) \\ 9(1.13 \%) & 14(2.24 \%)\end{array}$

$430(67.72 \%)$

$408(65.81 \%)$

$205(32.28 \%)$

$212(34.19 \%)$

$\begin{array}{ll}481(67.0 \%) & 397(64.24 \%) \\ 69(9.61 \%) & 61(9.87 \%) \\ 126(17.55 \%) & 123(19.90 \%) \\ 42(5.85 \%) & 37(5.99 \%)\end{array}$

$\chi^{2}=0.51 ; \mathrm{ns}$

$Z=2.05 ; \mathrm{p}<.05$

$\chi^{2}=3.32 ; \mathrm{ns}$

$42(5.85 \%)-37(5.99 \%)$

$$
\chi^{2}=6.49 ; \mathrm{ns}
$$

$387(80.46 \%) \quad 387(81.65 \%)$

$24(4.99 \%) \quad 23(4.85 \%)$

$6(1.25 \%) \quad 6(1.27 \%)$

$15(3.12 \%) \quad 26(5.49 \%)$

$49(10.19 \%) \quad 32(6.75 \%)$

Goals attained (0-3)

$\begin{array}{lll}2.79(.62) & 2.87(.64) & \mathrm{t}_{(1541)}=-2.4 ; \mathrm{p}<.05 \\ 2.73(.60) & 2.76(.66) & \mathrm{t}_{(1543)}=-1.0 ; \mathrm{ns} \\ 3.92(.79) & 3.98(.78) & \mathrm{t}_{(1527)}=-1.3 ; \mathrm{ns} \\ 3.02(.55) & 3.06(.61) & \mathrm{t}_{(1524)}=-1.2 ; \mathrm{ns} \\ 2.83(\mathrm{Mdn}) & 2.95(\mathrm{Mdn}) & Z=3.77 ; p<.05 \\ 1.32(\mathrm{Mdn}) & 1.38(\mathrm{Mdn}) & Z=1.06 ; \mathrm{ns} \\ 1.91(\mathrm{Mdn}) & 1.70(\mathrm{Mdn}) & Z=-4.43 ; p<.001\end{array}$

Note: SBCM-PRS: Psychiatric rehabilitation services with strengths-based case management; TAUPRS: Psychiatric rehabilitation services as treatment as usual. 
Table 2: Quality of life, unmet needs, symptom severity, and self-efficacy scores at baseline and follow-up, for the experimental $(\mathrm{N}=696)$ and control $(\mathrm{N}=580)$ groups $^{\dagger}$

\begin{tabular}{lccc}
\hline \multicolumn{1}{c}{ Measure } & Baseline & Follow-up & $\begin{array}{c}\text { experimental vs } \\
\text { control groups } \\
F(1,1252)\end{array}$ \\
\hline Quality of life (1-4) & $\mathrm{M}(\mathrm{SD})$ & $\mathrm{M}(\mathrm{SD})$ & $6.60^{* * *}$ \\
SBCM-PRS & $2.79(.62)$ & $2.89(.52)$ & \\
TAU-PRS & $2.87(.64)$ & $2.86(.56)$ & \\
& & & $4.71^{*}$ \\
Unmet needs (1-4) & & & \\
SBCM-PRS & $2.73(.60)$ & $2.83(.54)$ & \\
TAU-PRS & $2.76(.66)$ & $2.81(.57)$ & \\
& & & \\
Symptom severity (1-5) & & & \\
SBCM-PRS & $3.93(.79)$ & $4.08(.68)$ & \\
TAU-PRS & $3.98(.78)$ & $4.07(.68)$ & \\
& & & \\
Self-efficacy (1-5) & & & \\
SBCM-PRS & $3.02(.55)$ & $3.07(.49)$ & \\
TAU-PRS & $3.06(.61)$ & $2.97(.52)$ & \\
\hline$*$ & &
\end{tabular}
${ }^{*} p<.05 ;{ }^{* *} p<.01 ;{ }^{* * *} p<.001$.

Note: SBCM-PRS: Psychiatric rehabilitation services with strengths-based case management; TAUPRS: Psychiatric rehabilitation services as treatment as usual.

${ }^{\dagger}$ These models include gender, age and quality of life at baseline as covariates . Full models are obtainable from the authors upon request. 
Table 3: Change $\operatorname{scores}^{\mathrm{a}}(\Delta)$ for interpersonal relationships, goals set, goals attained, service utilization, quality of life domains, and unmet needs domains, for the experimental and control groups

\begin{tabular}{|c|c|c|c|c|c|}
\hline & \multicolumn{2}{|c|}{ SBCM-PRS } & \multicolumn{2}{|c|}{ TAU-PRS } & \multirow{2}{*}{$\begin{array}{c}\text { Univariate } \\
\text { statistics } \\
Z\end{array}$} \\
\hline & $\mathrm{n}$ & $\Delta \mathrm{M}(\mathrm{SD})$ & $\mathrm{n}$ & $\Delta \mathrm{M}(\mathrm{SD})$ & \\
\hline Interpersonal relationships (1-4) & 592 & $-.18(.60)$ & 566 & $-.30(.67)$ & $-4.44^{* * * *}$ \\
\hline Goals set $^{\mathrm{b}}(0-3)$ & 696 & $.42(1.52)$ & 580 & $.16(1.41)$ & $-3.29^{* * *}$ \\
\hline Goals attained $(0-3)$ & 375 & $.05(1.06)$ & 318 & $.45(1.06)$ & $-4.69^{* * *}$ \\
\hline Service utilization ${ }^{c}(1-4)$ & 245 & $-1.04(.77)$ & 263 & $.02(.63)$ & $-14.37^{* * *}$ \\
\hline \multicolumn{6}{|l|}{ Quality of life domains (1-4) } \\
\hline Physical health & 591 & $.03(.83)$ & 566 & $-.11(.89)$ & $-3.13^{* *}$ \\
\hline Leisure & 369 & $.12(1.03)$ & 331 & $-.10(1.01)$ & $-4.83^{* * *}$ \\
\hline Housing & 593 & $.09(.79)$ & 568 & $.02(.87)$ & $-2.01^{*}$ \\
\hline Employment & 217 & $.12(0.89)$ & 242 & $-.01(0.94)$ & $-2.55^{* *}$ \\
\hline Income & 587 & $.16(1.04)$ & 563 & $.12(1.06)$ & -0.65 \\
\hline Study & 26 & $.12(1.03)$ & 14 & $-.14(.86)$ & -1.08 \\
\hline \multicolumn{6}{|l|}{ Unmet needs domains (1-4) } \\
\hline Leisure & 568 & $-.39(1.53)$ & 555 & $-.51(1.58)$ & -1.65 \\
\hline Housing & 580 & $.13(1.07)$ & 567 & $.09(1.19)$ & -.78 \\
\hline Social & 588 & $.13(1.21)$ & 562 & $.09(1.19)$ & -.56 \\
\hline Employment & 70 & $-.16(1.18)$ & 49 & $-.12(1.26)$ & -.59 \\
\hline Income & 589 & $.05(.98)$ & 561 & $.01(1.08)$ & -1.05 \\
\hline Study & 26 & $-.27(.92)$ & 15 & $-.13(.83)$ & -.31 \\
\hline
\end{tabular}

$* p<.05 . \quad * * p<.01 . * * * p<.001$.

${ }^{a}$ calculated as $2^{\text {nd }}$ assessment score minus $1^{\text {st }}$ assessment score

${ }^{\mathrm{b}}$ Since the focus of this analysis is change scores, participants who set no goals at baseline or followup were included in the analysis.

${ }^{c}$ only for participants who had started to use services at least 6 months before the beginning of the study (as described in the measures section).

Note: SBCM-PRS: Psychiatric rehabilitation services with strengths-based case management; TAUPRS: Psychiatric rehabilitation services as treatment as usual. 
Figure 1: Service users flow through the different stages of the study

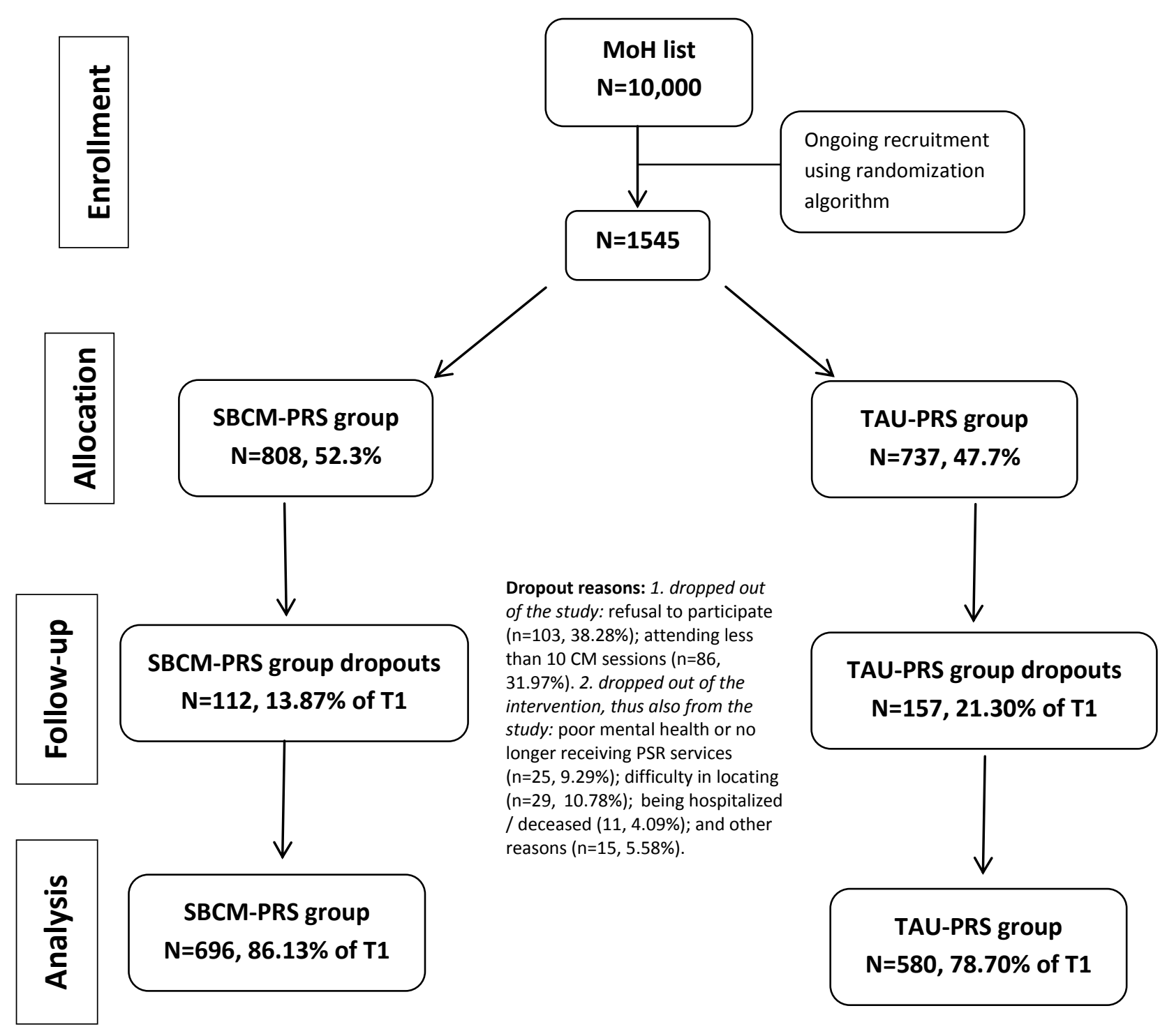

